\title{
CONTACT TRANSITIONS TRACKING DURING FORCE-CONTROLLED COMPLIANT MOTION USING AN INTERACTING MULTIPLE MODEL ESTIMATOR
}

\author{
Lyudmila MIHAYLOVA, Tine LEFEBVRE, Ernesto STAFFETTI, \\ Herman BRUYNINCKX and Joris De SCHUTTER
}

\begin{abstract}
1. Introduction
In different robot operations the manipulator has to interact with the environment through the manipulated object and modify its trajectory depending on the contact forces that arise. These force-controlled operations are called compliant motion tasks. Force control is required due to the fact that small errors in the models can generate high forces on the manipulator. For other tasks, such as cutting, welding or polishing, the robotic manipulator has to apply a given force to execute correctly the task. In all cases the manipulator is moving an object in contact with the environment through a sequence of contact configurations. In this paper the objects involved in the compliant motion are supposed to be rigid and polyhedral. The path of the manipulated object is a sequence of contact configurations. The configurations can be grouped into a subsequence of configurations equivalent from a topological point of view, i.e., in which the same elements of the manipulated object are in contact with the same elements of the environment. In this context each class of equivalence is called contact formation $(C F) .^{5}$
\end{abstract}

This work assumes uncertainties in the position and orientation of both the manipulated object and the environment. In practice, besides these model uncertainties other sources of uncertainties are present such as friction, sensor noises, geometrical uncertainies such as burrs, or unexpected events. The focus here is on the detection of the current $\mathrm{CF}$ and the instant of transition between the CFs.

INFORMATION \& SECURITY. An International Journal, Vol. 9, 2002, 114-129 
Encoders, mounted at the robot joints, supply information about the end-effector location and motion, and a force sensor, mounted at the robot wrist, gives information about the interaction with the environment. This information is also used to estimate the uncertain geometric parameters. In Bruyninckx et al. ${ }^{2}$ a possible architecture of an autonomous assembly system is proposed. It is pointed out that such a system needs a high-level planner (responsible for planning, re-planning and on-line error recovery), a low-level module (responsible for sensing and the execution of the planned action), and a medium-level module (for estimation and monitoring).

This work presents and generalizes results reported earlier. ${ }^{9}$ The possible CFs are described by different models and, with them, an Interacting Multiple Model (IMM) estimator is implemented. Its performance is investigated and evaluated by experiments with real data of different type: velocities and forces. Other works treat forcecontrolled compliant motion tasks. ${ }^{3,4,7}$ Thus, the problem of estimating first-order geometric parameters and monitoring contact transitions has been approached through single-model Extended Kalman Filters (EKFs), run in parallel for the known different CFs. ${ }^{3}$ The Summed Normalized Innovation Squared (SNIS) test has been used as an indicator of the transitions between the CFs. One solution to the estimation of the geometric parameters for one CF was proposed on the basis of iterated EKF. ${ }^{7,8}$

The remaining parts of this article are organized as follows. In section 2 the problem of contact transitions' tracking during force-controlled compliant motion is formulated as a state estimation problem of hybrid systems. Section 3 gives the state and measurement equations of a compliant motion with subsequent CFs, namely those of moving a cube into a corner. Section 4 describes an Interacting Multiple Model estimator and its connection with the planning part. Section 4 yields performance analysis for the cubein-corner assembly with experimental data involving a KUKA-IR 361 robot. The final section provides concluding remarks. Short guiding rules for the Jacobian matrices computations of the measurement and closure equations are given in the Appendix.

\section{Problem formulation}

During the compliant motion different CFs occur. They can involve, for instance, a contact between an edge of the manipulated object and a face of the environment (edgeface contact), a face of the manipulated object and a face of the environment (face-face contact), and so on (Figure 1). To estimate the unknown geometric parameters and track the transitions between CFs, the manipulated object and the environment are considered as a stochastic hybrid system with continuous and discrete uncertainties. The state-space equations are of the form

$$
x_{k+1}=f\left(x_{k}, m_{k}\right)+g\left(m_{k}, \eta_{k}\right),
$$




$$
h_{k}\left(x_{k}, m_{k}, z_{k}, \xi_{k}\right)=0,
$$

where $x_{k} \in \mathbb{R}^{n_{x}}$ is the system state vector, estimated based on the measurement vector $z_{k} \in \mathbb{R}^{n_{z}} ; m_{k}$ is the modal state, corresponding to the CF. The measurement equation is in implicit form, ${ }^{3,10}$ in which $h_{k}$ is a function of both the estimated variables and the measured data $z_{k}$. The additive system and measurement noises $\eta_{k} \in \mathbb{R}^{n_{\eta}}$ and $\xi_{k} \in \mathbb{R}^{n_{\xi}}$ are mutually independent, white with zero mean and covariances $Q_{k}$ and $R_{z, k}$, respectively. The functions $f, g$ and $h$ are nonlinear and remain unchanged during the estimation procedure.

In this paper the focus is on the detection of the current $\mathrm{CF}$ and the instant of transition between the CFs. It is supposed that the changes between the CFs are modeled by a first-order Markov chain with initial and transition probabilities, respectively

$$
\begin{gathered}
P\left\{m_{j, 0}\right\}=\mu_{j}(0), \\
P r\left\{m_{j, k+1} / m_{i, k}\right\}=\pi_{i j, k},
\end{gathered}
$$

where

$$
\sum_{j=1}^{N} \pi_{i j, k}=1, i=1, \ldots, N
$$

and $\pi_{i j, k}$ is the transition probability from $\mathrm{CF} m_{i}$ to $\mathrm{CF} m_{j}$. At the same time, the unknown geometric parameters of the manipulated object and of the environment are estimated.

The solution to the state estimation problem with unknown model (1)-(2) can be provided by Bayesian sub-optimal MM estimators, between which the Interacting Multiple Model (IMM) filter has proven to be one of the most efficient schemes. Within the framework of the MM estimation the lack of knowledge about the exact model is replaced by a discrete set of models $\mathbb{M} \triangleq\left\{m_{1}, m_{2}, \ldots, m_{N}\right\}$, each of them describing possible modes/regimes, here different CFs. With the models several Kalman filters are run in parallel. The IMM estimator calculates the state estimate as a probabilistically weighted sum of the state estimates $\hat{x}_{j, k / k}$ from the Kalman filters with the mode probabilities $\mu_{j, k}$, namely ${ }^{1}$

$$
\hat{x}_{k / k}=\sum_{p_{j, k} \in \mathbb{M}} \hat{x}_{j, k / k} \mu_{j, k}
$$

and the associated covariance matrix accordingly.

Usually the constructed models for the unknown system modes/ regimes are multiple system models (1). This paper considers tracking task, where the modes, i.e. the CFs 


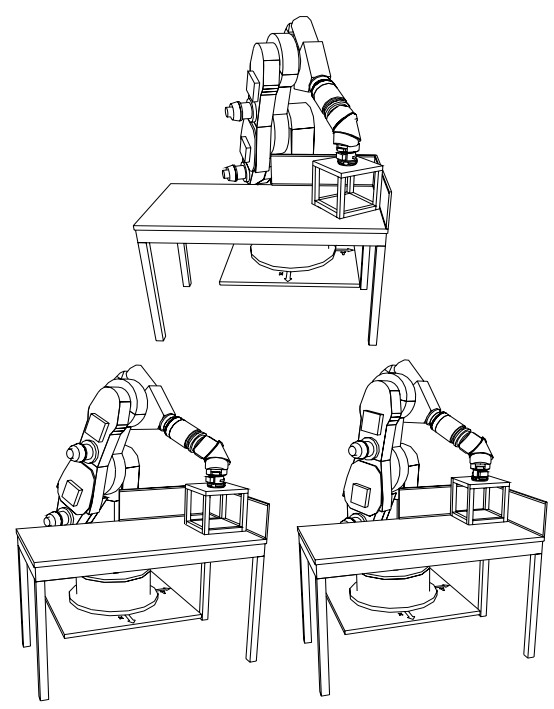

Figure 1: Robot placing a cube in a corner.

are described through several nonlinear measurement models (2), subject to nonlinear kinematic constraints, called closure equations. ${ }^{3,8}$

\section{State and measurement equations}

The system equation describes the positions and orientations of the manipulated object and the environment and it is linear.

State equation. The system model is of the form

$$
x_{k+1}=x_{k}+\eta_{k} .
$$

The estimated states are geometric grasping and environment parameters (positions and orientations). The state vector $x_{k}=\left(x_{k}^{m o^{T}}, x_{k}^{e n v^{T}}\right)^{T}$ comprises a part $x_{k}^{m o}=$ $\left(x_{k}^{m}, y_{k}^{m}, z_{k}^{m}, \theta_{x, k}^{m}, \theta_{y, k}^{m}, \theta_{z, k}^{m}\right)^{T}$, referring to the manipulated object, and a part $x_{k}^{e n v}=$ $\left(x_{k}^{e}, y_{k}^{e}, z_{k}^{e}, \theta_{x, k}^{e}, \theta_{y, k}^{e}, \theta_{z, k}^{e}\right)^{T}$, referring to the environment. These positions and orientations do not change during the task execution, i.e. the states are static. Four reference frames are considered (Figure 2): $\{\mathrm{w}\}$ is the world frame, $\{\mathrm{g}\}$ is a frame on the gripper of which the position and orientation with respect to the world frame $\{w\}$ are exactly known (through the position kinematics of the robot), $\{\mathrm{m}\}$ is a frame fixed to 
the manipulated object, $\{\mathrm{e}\}$ is a frame fixed to the environment. $x_{k}^{m o}$ are considered with respect to $\{\mathrm{g}\}$, and $x_{k}^{e n v}$ relative to $\{\mathrm{w}\}$.

Measurement equation. The sensor measurements are translational and angular endeffector velocities, $v_{k}$ and $\omega_{k}$, together with contact forces and moments, $f_{k}$ and $m_{k}$, measured by a force/torque sensor. They are grouped in the twist $t_{k}=\left(v_{k}^{T}, \omega_{k}^{T}\right)^{T}$, wrench $w_{k}=\left(f_{k}^{T}, m_{k}^{T}\right)^{T}$ and measurement $z_{k}=\left(t_{k}^{T}, w_{k}^{T}\right)^{T}$ vectors. Measurement equations are derived for each $\mathrm{CF}$ from the reciprocity condition. ${ }^{3}$

This condition states that any twist of the manipulated object is reciprocal to any wrench of the modeled wrench space (spanned by the basis $G_{i}$ ) and that any wrench is reciprocal to any twist of the modeled twist space (spanned by the basis $J_{i}$ ). Index $i$ refers to the $i$-th CF. Then Eq. (2) acquires the form

$$
h_{i, k}=\left(\begin{array}{l}
G_{i, k}^{T}\left(x_{i, k}\right) t_{k} \\
J_{i, k}^{T}\left(x_{i, k}\right) w_{k}
\end{array}\right)=0 .
$$

Both $G_{i}$ and $J_{i}$ contain trigonometric functions (sines and cosines) of the estimated states, such that the measurement functions ${ }^{8}$ are nonlinear. To every CF correspond different twist and wrench bases. The models in Eq. (7) are very distinct, which is appropriate for using the multiple-model approach to solve the problem. Equation (7)

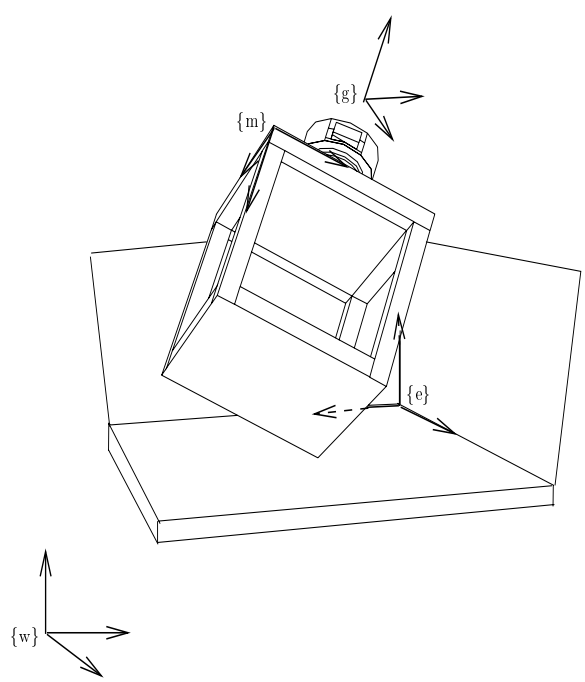

Figure 2: Frames. 
is linearized for each CF around the current predicted state estimate $\hat{x}_{i, k+1 / k}$. For the computation of the derivative of $h_{i, k}($.$) with respect to the estimated variables, the$ partial derivatives of $J_{i, k}$ and $G_{i, k}$ are needed (See the Appendix).

Closure equations. The occurrence of a CF yields additional information for the state variables. The so-called kinematic closure equations ${ }^{3}$

$$
c_{i}\left(x_{i, k}\right)=0
$$

describe additional nonlinear constraints that relate different configuration variables (of the manipulated object and the environment) for each CF. The closure equations are models of the contacts obtained as a composition of basic contacts (vertex-face and edge-edge) between polyhedral objects. For instance, the edge-face contact between the cube and the environment is described by means of two vertex-face contacts, the contact between two faces of the object is described as a composition of three vertexface contacts, and so on.

For each $\mathrm{CF}$, the closure equation is applied once. Its corresponding EKF uses as initial state estimate and covariance matrix the ones obtained from the EKF based on the measurement equation. The state estimate and its covariance matrix, computed by the closure equations, are given to the interacting step of the IMM algorithm.

\section{IMM estimator for transition and CF monitoring}

The number of possible CFs between the manipulated object and the environment is generally high. ${ }^{5}$ A set of mutually exclusive and exhaustive hypotheses is constructed to describe all possible CFs of the manipulated object from one place to another. For the case in which the manipulated object is a cube and the environment is a corner this number is $249 .{ }^{12}$ In the planner, ${ }^{12}$ a graph is constructed so that its nodes correspond to the possible CFs and its arcs to the transitions between them. Given the path of the motion and the level of uncertainty about the geometric parameters, it is possible to eliminate from the set of hypotheses those CFs whose distance $d$ from the nodes of the path is higher than a given threshold $d_{\max } \cdot{ }^{13}$ The distance $d$ is the minimum number of arcs of the CF graph that are between two nodes. In this way, a relevant amount of CFs can be eliminated ${ }^{2,5}$ and the number of hypotheses considerably reduced. Here it is assumed that the hypothesis $H_{0}$ corresponds to the case of completely constrained object. Hypotheses $H_{i}, i=1, \ldots, N$ describe all other CFs. With the models for each $\mathrm{CF}$ and its EKFs, an IMM estimator is implemented. So, the CFs can be monitored on-line, using the information provided by the IMM mode probabilities.

The nonlinear character of the measurement equations requires the use of EKFs or other nonlinear filtering techniques that do not require computation of derivatives. ${ }^{6}$ 
The present work estimates the state vectors through EKFs. Each EKF is of the form

$$
\begin{aligned}
& \hat{x}_{i, k+1 / k+1}=\hat{x}_{i, k+1 / k}+K_{i, k+1} \nu_{i, k+1}, \\
& \hat{x}_{i, k+1 / k}=\hat{x}_{i, k / k}, \\
& P_{i, k+1 / k}=P_{i, k / k}+Q_{i, k}, \\
& K_{i, k+1}=-P_{i, k+1 / k} H_{x_{i}, k+1}^{T} S_{i, k+1}^{-1}, \\
& P_{i, k+1, k+1}=\Gamma_{i, k+1} P_{i, k+1 / k} \Gamma_{i, k+1}^{T}+K_{i, k+1} R_{i, k+1} K_{i, k+1}^{T}, \\
& S_{i, k+1}=R_{i, k+1}+H_{x_{i}, k+1} P_{i, k+1 / k} H_{x_{i}, k+1}^{T},
\end{aligned}
$$

where

$$
\begin{aligned}
\Gamma_{i, k+1} & =I+K_{i, k+1} H_{x_{i}, k+1}, \\
R_{i, k+1} & =D_{i, k+1} R_{z, k+1} D_{i, k+1}^{T}, \\
H_{x_{i}, k+1} & =\partial h_{i} / \partial \hat{x}_{i, k+1 / k}, D_{i, k+1}=\partial h_{i} / \partial z_{k+1}, \\
\nu_{i, k+1} & =h_{i}\left(\hat{x}_{i, k+1 / k}, z_{k+1}\right) .
\end{aligned}
$$

$\hat{x}_{i, k+1 / k+1}$ and $\hat{x}_{i, k+1 / k}$ are, respectively, the filtered and predicted state vectors, $K_{i, k+1}$ is the EKF gain matrix, $P_{i, k / k}$ is the estimation error covariance matrix, $\nu_{i, k+1}$ is a "pseudo-innovation" process ${ }^{10}$ and $S_{i, k+1}$ - its covariance matrix. $I$ denotes the identity matrix.

The Appendix presents guiding rules for the computation of the Jacobian matrices for the measurement and closure equations. Lefebvre and coauthors provide detailed derivation of the measurement models (models of different $\mathrm{CFs}$ ). ${ }^{8}$

\section{Performance analysis on a cube-in-corner assembly}

The proposed approach is applied to a cube-in-corner assembly system (Figure 1). The experimental data are obtained with a KUKA-IR 361 industrial robot. The cube is mounted directly on the robot without flexibility between them. The measurements are taken at a frequency of $10 \mathrm{~Hz}$. The experimental data (Figures 3-6) correspond to the three CFs of the cube-in-corner assembly (Figure 1): $k \in[0,220]$ is the face-face and edge-face contact, $k \in[221,450]$ is the two face-face contact, and $k \in[450,545]$ is the three face-face contact (completely constrained case). In the test a path with three $\mathrm{CFs}$ is used. In the IMM the hypotheses are: $H_{0}$ - three face-face contact, $H_{1}$ - two faceface contact, $H_{2}$ - face-face and edge-face contact. The noise covariance matrices $Q$ and $R_{z}$

$$
\begin{gathered}
Q=\operatorname{diag}\{5,5,5,0.001,0.001,0.001,1,1,1,0.001,0.001,0.001\} \\
R_{z}=\operatorname{diag}\left\{0.05,0.4,1.96,4 \cdot 10^{-7}, 5 \cdot 10^{-6}, 9 \cdot 10^{-8}, 0.06,0.009,0.008,150,87,51\right\}
\end{gathered}
$$




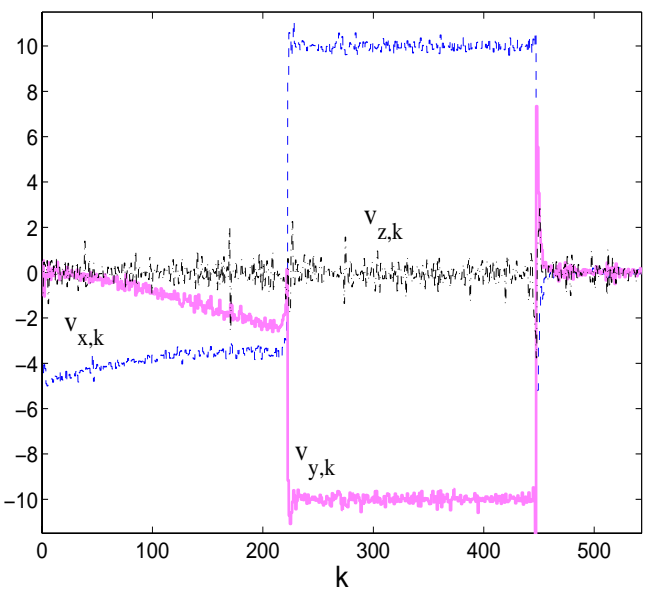

Figure 3: Measured translational velocities.

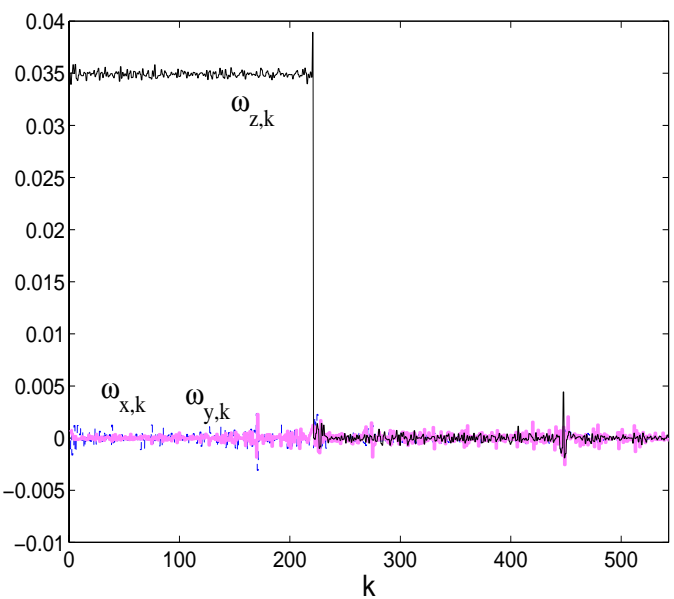

Figure 4: Measured angular velocities.

are the same for all EKFs. The units of the elements of $Q$ are $\mathrm{mm}^{2}$ and $\mathrm{rad}^{2}$, respectively for the positions and angles, and those of $R_{z}$ are $(\mathrm{mm} / \mathrm{sec})^{2},(\mathrm{rad} / \mathrm{sec})^{2}, N^{2}$, $(N \mathrm{~mm})^{2}$ for the measured velocities, forces and moments. The system noise covari- 


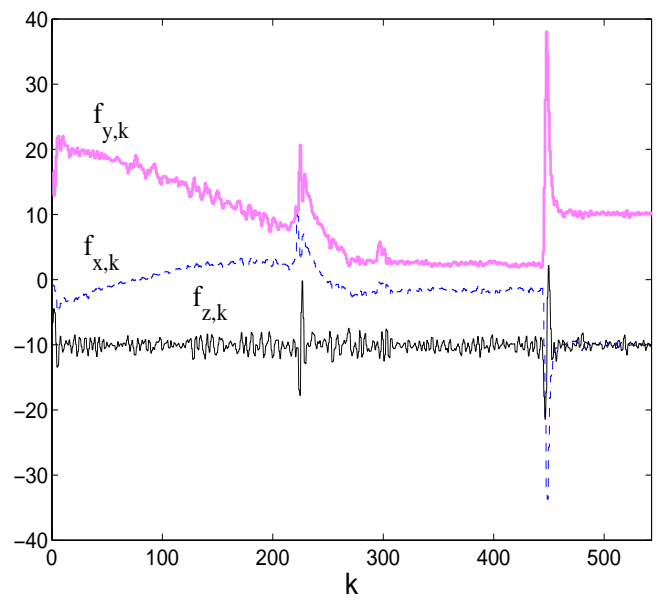

Figure 5: Measured forces.

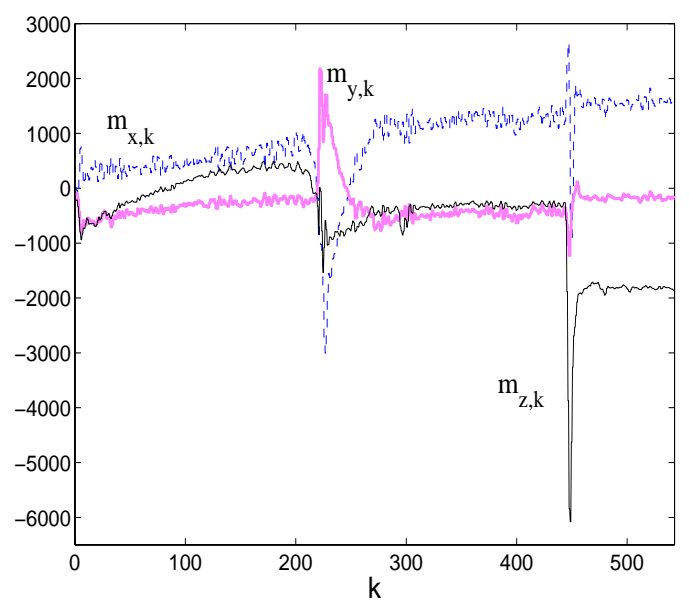

Figure 6: Measured moments.

ance matrix $Q$ reflects the presence of linearization errors, whereas the measurement noise covariance for the used sensors is known. The IMM transition probability matrix 


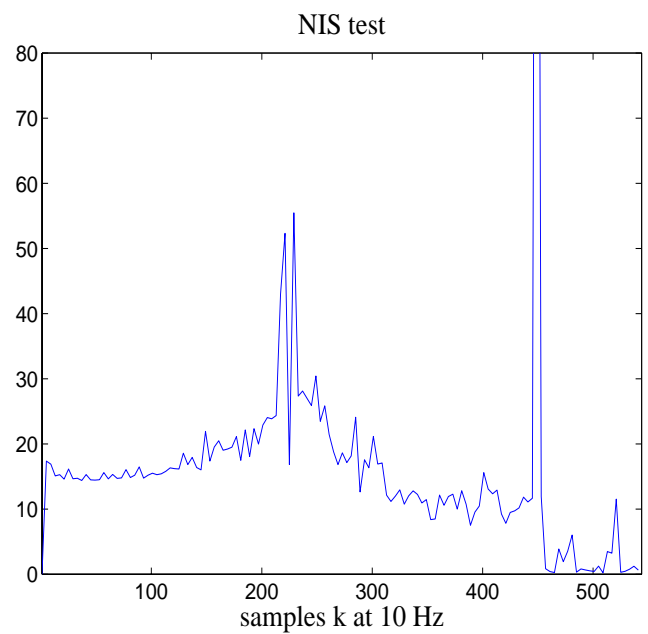

Figure 7: Normalized Innovation Squared test.

and the initial probability vector are chosen as follows:

$$
\operatorname{Pr}=\left(\begin{array}{lll}
0.98 & 0.01 & 0.01 \\
0.01 & 0.98 & 0.01 \\
0.01 & 0.01 & 0.98
\end{array}\right), \mu_{0}=\left(\begin{array}{l}
1 / 3 \\
1 / 3 \\
1 / 3
\end{array}\right)
$$

Due to a lack of information, equal initial probabilities are assigned to all CFs.

It is obvious from Figure 8 that, based on the IMM probabilities, the contact transitions can be detected on time. After the change a small period is needed and the algorithm resolves the "competition" between the CFs. This is reflected also in the Normalized Innovation Squared (NIS) test, ${ }^{1} \epsilon_{k}=\nu_{k} S_{k}^{-1} \nu_{k}$ (Figure 7) and in the peak estimation errors. In the periods of transitions the estimates are not reliable. The estimation error $e_{k}=\hat{x}_{k / k}-x_{k}$ of the positions and orientations is presented in Figures 9-12. The NIS test (Figure 7) and the mode probabilities (Figure 8) contain information about the type and instants of contact transitions. By the IMM approach the CFs and the transitions between them are detected on-line and, at the same time, the unknown parameters of the manipulated object and the environment are estimated. So, both modes detection and estimation are performed automatically. In earlier works of the research team 7,8 the detection of the CFs was performed from the information of the SNIS test of independently working EKFs and their residual errors. Of course, the computational cost is proportional to the number of the EKFs (the number of CFs). The IMM filter, implemented in the present paper, is with a fixed structure, i.e. with preliminary 


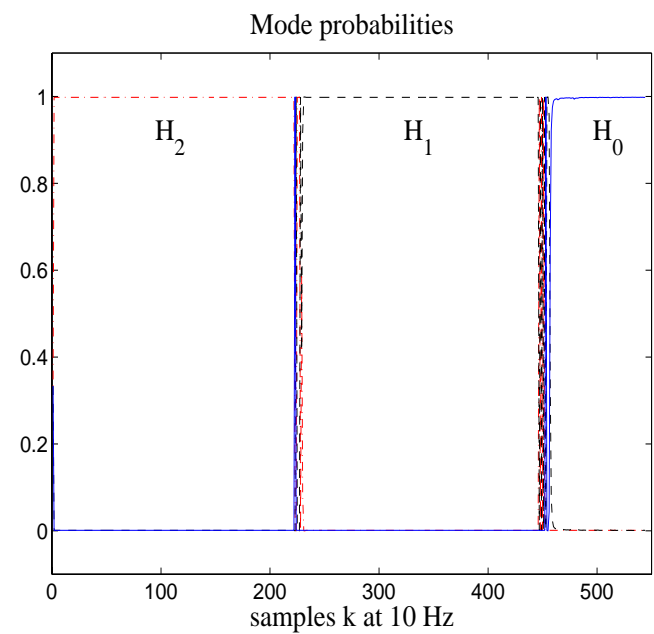

Figure 8: IMM mode probabilities.

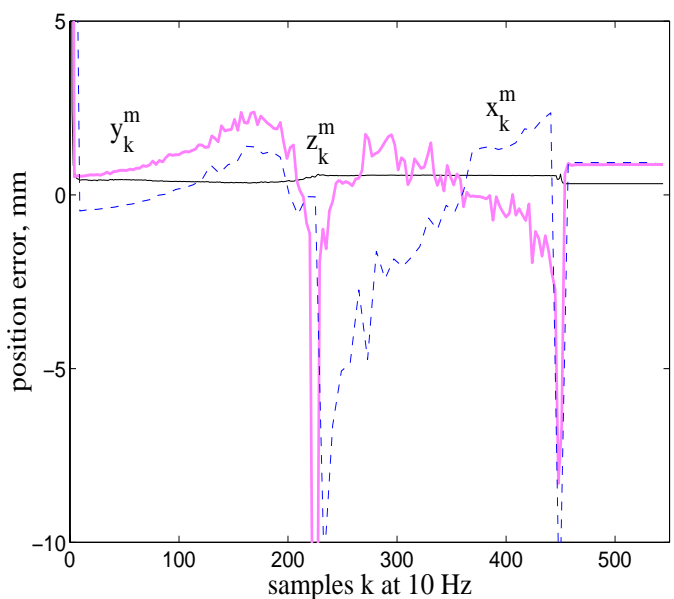

Figure 9: Error $e_{k}$ in positions of the cube.

determined set of models. When the estimation block is connected with the planning part, ${ }^{5,12,13}$ the estimator can receive from the graph of the planner information about the next neighboring CFs. Based on this graph structure of the CFs, variable structure 


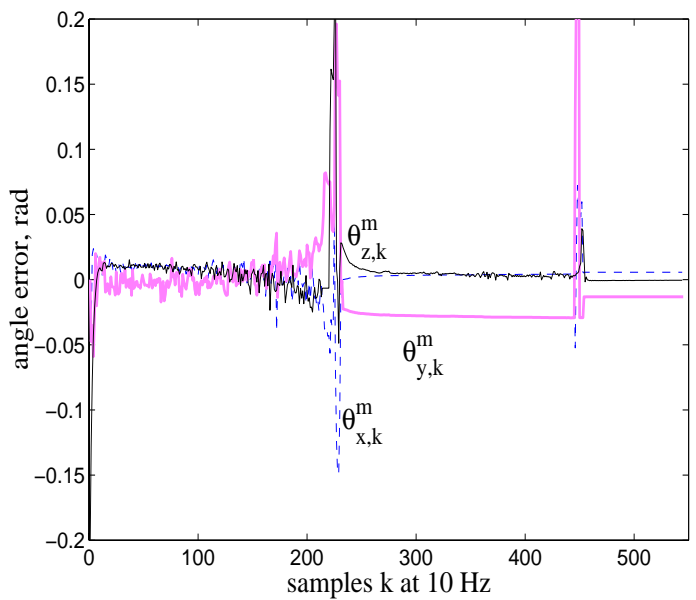

Figure 10: Error $e_{k}$ in orientation angles of the cube.

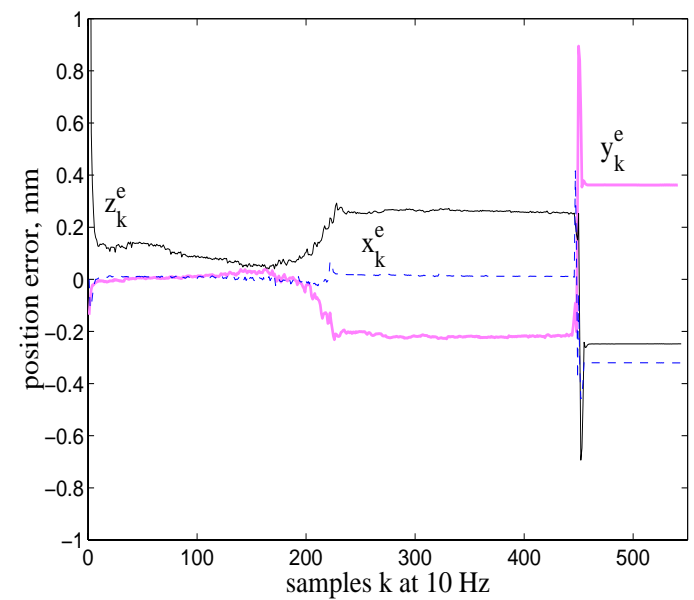

Figure 11: Error $e_{k}$ in positions of the environment.

IMM estimators (with time varying set of models) can be designed.

Extensions to cases with time-varying geometric parameters of the manipulated object and the environment can be performed by analogy. 


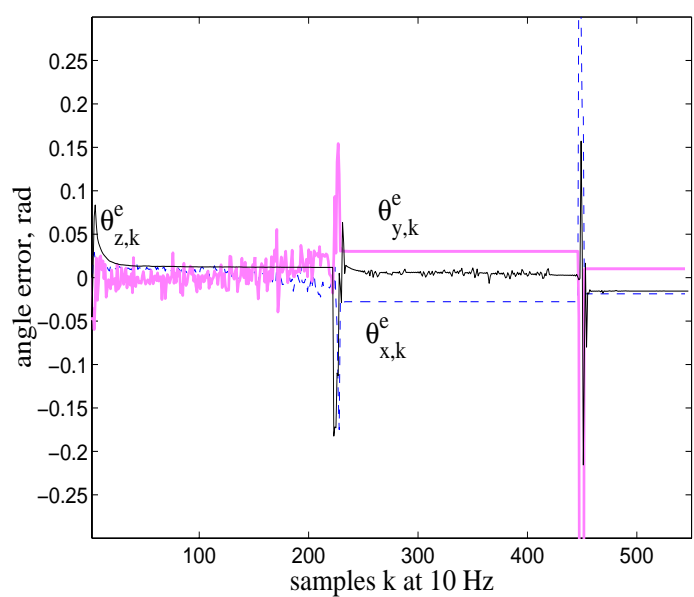

Figure 12: Error $e_{k}$ in angles of the environment.

\section{Conclusions}

In this paper a general approach to contact transitions detection and estimation of uncertain geometric parameters (positions and orientation angles) is proposed for forcecontrolled robotic tasks in which a robotic manipulator moves an object in contact with the environment, both rigid and polyhedral.

The possible CFs are described by different measurement equations, whereas the system equation is known. An IMM estimator is implemented and its performance is evaluated by real sensor data (linear and angular velocities, forces and moments). The IMM probabilities and the normalized innovation squared test permit to monitor the occurring CFs. The experimental assembly of moving a cube into a corner demonstrates high estimation accuracy and quick detectability of the contact transitions.

\section{Appendix. Derivatives Computation}

The computation of the measurement and closure equations is based on the screwtransformation matrices, ${ }^{3}$ that are functions of the rotational matrices between the different frames. ${ }^{3,4}$ The partial derivatives are found from Eq. (7) and have the form

$$
\frac{\partial h_{i, k}}{\partial x_{i, k}}=\left(\begin{array}{ll}
\partial\left(G_{i, k}^{T}\left(x_{i, k}\right)\right) / \partial x_{i, k}^{m T} & \partial\left(G_{i, k}^{T}\left(x_{i, k}\right)\right) / \partial x_{i, k}^{e T} \\
\partial\left(J_{i, k}^{T}\left(x_{i, k}\right)\right) / \partial x_{i, k}^{m T} & \partial\left(J_{i, k}^{T}\left(x_{i, k}\right)\right) / \partial x_{i, k}^{e T}
\end{array}\right),
$$




$$
D_{i, k}=\partial h_{i, k} / \partial z_{k}=\left(\begin{array}{cc}
G_{i, k}^{T} & 0 \\
0 & J_{i, k}^{T}
\end{array}\right)
$$

The matrix derivatives are computed according to the rules for matrix calculus operations proposed by Vetter. ${ }^{11}$

\section{Acknowledgement}

Herman Bruyninckx and Tine Lefebvre are, respectively, Postdoctoral and Doctoral Fellows of the Fund for Scientific Research-Flanders (F.W.O-Vlaanderen) in Belgium. Lyudmila Mihaylova is a Postdoctoral Fellow at Katholieke Universiteit Leuven, on leave from the Bulgarian Academy of Sciences. Financial support by the Center of Excellence BIS21 grant ICA1-2000-70016 and the K. U. Leuven's Concerted Research Action GOA/99/04 are gratefully acknowledged.

\section{Notes:}

1 Yaakov Bar-Shalom and Xiao-Rong Li, Estimation and tracking: Principles, techniques and software (Boston, MA: Artech House, 1993).

${ }^{2}$ H. Bruyninckx, T. Lefebvre, L. Mihaylova, E. Staffetti, J. De Schutter and J. Xiao, "A roadmap on autonomous robotic assembly," in Proceedings of the International Symposium on Assembly and Task Planning (Fukuoka, Japan, 2001), 49-54.

3 J. De Schutter, H. Bruyninckx, S. Dutré, J. De Geeter, J. Katupitiya and T. Lefebvre, "Estimating first-order geometric parameters and monitoring contact transitions during force-controlled compliant motions," Int. J. Robotics Res. 18, 12 (1999): 1121-1184.

4 J. De Schutter and H. Van Brussel, "Compliant robot motion, I, II," Int. J. Robotics Res. 7, 4 (1988): 3-33.

5 X. Ji and J. Xiao, "Automatic generation of high-level contact state space," in Proc. of the 1999 IEEE International Conf. on Robotics and Automation (1999), 238-244.

6 S. Julier, J. Uhlman and H. Durran-White, "A new method for the transformation of means and covariances in filters and estimators," IEEE Trans. on Aut. Control 45, 3 (2000): 477-482.

7 T. Lefebvre, H. Bruyninckx and J. De Schutter, "Estimation and propagation of uncertainties during force-controlled execution of contact formation sequences," in Proc. of the 10th Int. Conf. on Advanced Robotics (Budapest, Hungary, 2001), 85-90.

8 T. Lefebvre, H. Bruyninckx and J. De Schutter, "Polyhedral contact formation modeling and identification for autonomous compliant motion," IEEE Trans. on Automation and Robotics (2003). - To appear.

9 L. Mihaylova, T. Lefebvre, H. Bruyninckx, E. Staffetti and J. De Schutter, "Tracking contact transitions during force-controlled compliant motion using an Interacting Multiple Model Estimator," in Proc. of the 10th International Conf. on Advanced Robotics (Budapest, Hungary, 2001), 665-670. 
10 S. Soatto, R. Frezza and P. Perona, "Motion estimation via dynamic vision," IEEE Trans. on Aut. Control 41, 3 (1996): 393-414.

11 W. Vetter, "Matrix calculus operations and Taylor Expansions," SIAM Review 51 (1973): 352-369.

$12 \mathrm{~J}$. Xiao and X. Ji, "A Divide-and-merge approach to automatic generation of contact states and planning of contact motions," in Proc. of the IEEE Intern. Conf. on Robotics and Automation (2000).

13 J. Xiao and L. Zhang, "Towards obtaining all possible contacts - growing a polyhedron by its location uncertainty," IEEE Trans. on Robotics and Automation 12, 4 (1996): 553-565.

LYUDMILA MIHAYLOVA received the M.S. and Ph.D. degrees in Systems and Control Engineering from the Technical University of Sofia, Bulgaria, in 1989 and 1996 respectively. Since 1996 she is a Research Associate at the Bulgarian Academy of Sciences. From September 2000 till October 2002 she has been a Postdoctoral Fellow at the Katholieke Universiteit Leuven, Belgium, Department of Mechanical Engineering, involved in the development and application of advanced techniques for filtering and estimation of nonlinear stochastic systems under uncertainties, machine learning, and active sensing. Her other interests are in the areas of system identification and control and sensor data fusion. Dr. L. Mihaylova is a member of the IEEE. E-mail: Lyudmila.Mihaylova@mech.kuleuven.ac.be. 
TINE LEFEBVRE received her Masters degree in mechanical engineering, specialization in Mechatronics, from the Katholieke Universiteit Leuven, Belgium, in 1999. She is currently $\mathrm{PhD}$ student in Applied Sciences at the same university, with the Fund for Scientific ResearchFlanders (F.W.O) in Belgium. In 2000, she visited the Robotics Lab at Stanford University for three months. Her research focuses on modeling, non-linear estimation and active sensing for the on-line estimation of localization uncertainties in sensor-based robot tasks.

ERNESTO STAFFETTI received the M.S. degree in Electronic Engineering in 1995 from the Università degli Studi di Roma "La Sapienza," Rome, Italy, and Ph.D. degree in Electronic Engineering from the Universitat Politècnica de Catalunya, Barcelona, Spain, in 2002. He was Research Fellow at the Dipartimento di Informatica e Sistemistica of the Università degli Studi di Roma "La Sapienza," Research Associate at the Universitat Politècnica de Catalunya and at the Department of Mechanical Engineering of the Katholieke Universiteit Leuven, Heverlee, Belgium. Currently, he is a Research Associate at the Consejo Superior de Investigaciones Científicas, at the Institut de Robòtica i Informàtica Industrial, Barcelona, Spain. His interests are in the fields robot motion planning, compliant motion and kinematics of robot manipulators.

HERMAN BRUYNINCKX obtained the Bachelor's degree in Mathematics in 1984, M.Sc. degree in Computer Science in 1987 and in Mechatronics (1988), all from the Katholieke Universiteit Leuven, Belgium. In 1995, he received his PhD degree in Applied Sciences from the same university. Since 1994 he is a Post-Doctoral Researcher with the Fund for Scientific ResearchFlanders (F.W.O) in Belgium, and since 1995 he is an Assistant Professor at the Department of Mechanical Engineering, Katholieke Universiteit Leuven. His research interests include on-line estimation in sensor-based robot tasks, kinematics of robots, geometric foundations of robotics compliant robot motion, force control, active sensing, and motion planning. Dr. Bruyninckx is a member of the IEEE.

JORIS DE SCHUTTER received the degree of mechanical engineer from the Katholieke Universiteit Leuven, Belgium, in 1980, the M.S. degree from the Massachusetts Institute of Technology, in 1981, and the Ph.D. degree in Mechanical Engineering in 1986 from the Katholieke Universiteit Leuven. In 1986, he became a Lecturer in the Department of Mechanical Engineering, Division PMA (Production, Machine Design and Automation), Katholieke Universiteit Leuven, where he is a Professor since 1995. He teaches courses in kinematics and dynamics of machinery, control and robotics. His other research interests are in force control, compliant motion, position control of flexible systems and drive systems, and data fusion. Prof. De Schutter is a member of the IEEE. 